do you have a number of involvements with someone?". Support expectation was measured by "Can you expect to get a support by each of families or relatives, friends, and formal services?".

Results: Statistically significant differences were found between social isolation and support expectation. When the support could be expected from "families and relatives", "friends" who live inside or outside of the community, a number of involvements were found significantly higher average $(\mathrm{P}<.05)$. Between the expectation for formal services and a number of involvements were not found a significant difference.

Conclusion: These results are suggested that support expectations for daily lives are given up by social isolation in the elderly. Social isolation can be a risk of life continuation in the marginal community.

\section{WALKING SPEED OF OLDER PEOPLE AND PEDESTRIAN CROSSING TIME}

E. Duim ${ }^{1}$, M. Lebrao ${ }^{1}$, Y. Duarte ${ }^{2}$, J.F. Antunes ${ }^{1}$, 1. Public Health School, University of Sao Paulo, Sao Paulo, Brazil, 2. Nursing School, University of Sao Paulo, Sao Paulo, Brazil

Decreased gait speed in older people may put them at risk of injury and social seclusion for being unable to cross streets at safe. Traffic lights consider the walking speed of 1.1 meters per second $(\mathrm{m} / \mathrm{s})$ to regulate the time for pedestrian crossing in Sao Paulo, Brazil as in New York City. However, this regulation does not consider walking speed changes across time.

The objective was to assess walking speed of older people, and to compare these results with international standards for pedestrian crossing. In this cross-sectional study, the probabilistic sample of 1,191 individuals aged 60 or more years, in the city of Sao Paulo, Brazil, in 2010. Walking speed was directly assessed by a physical test, and dichotomously classified using two cut-off points: 1.1 and $0.9 \mathrm{~m} / \mathrm{s}$. Interviews informed sociodemographic and health status characteristics. A preponderant proportion $(95.7 \%)$ of older adults walks at a slower pace than is currently demanded pedestrian lights $(1.1 \mathrm{~m} / \mathrm{s})$. Reducing the reference speed to $0.9 \mathrm{~m} / \mathrm{s}$ would allow decreasing this proportion to $69.7 \%$. Women, brown individuals, those less schooled and those reporting poorer health status had higher odds of walking at a slower pace than would be required by traffic lights to cross streets in the city. Most older adults cannot cross streets at their own walking speed in São Paulo. These results reinforce the need of interventions targeting the whole traffic environment, to prevent accidents with older people and to promote urban mobility.

\section{RESIDENTS' CHANGE IN SOCIALIZATION AND} LONELINESS SINCE MOVING TO SENIOR HOUSING H. Taylor, S. Herbers, N.L. Morrow-Howell, Washington University in St. Louis, St. Louis, Missouri

It is frequently assumed older adults who move to senior housing communities (SHCs) will socialize more and be less lonely. The purpose of this study is to describe changes in residents' frequency of socialization and loneliness since moving into senior housing, and to determine factors associated with these changes. Ninety-six older adults living in two SHCs completed cross-sectional surveys with items regarding changes in socialization and loneliness since moving into a SHC, age, education, depressive symptoms, anxiety symptoms, years lived in the SHC, and distance to previous residence. Analyses for the current study include descriptive statistics and multinomial logistic regression models. Since moving into a SHC, twentyfive percent of residents had decreases in socializing with family, 20\% had decreases in socializing with friends, $19 \%$ had decreases in level of companionship, $21 \%$ felt more left out, and $14 \%$ felt more isolated. Age, education, anxiety symptoms, and years living in the SHC were related to changes in socialization and loneliness in the multinomial logistic models. These analyses suggest a significant portion of older adults living in senior housing may socialize with friends and family less and feel lonelier after moving to senior housing. It is important to understand the reasons for decreasing social interactions and increasing loneliness after older residents move to senior housing, and how to intervene upon their behalf. Additional studies are needed to determine if these findings are consistent, and interventions should be developed to increase socializing and decrease loneliness among residents living in senior housing.

\section{THE SIGNIFICANCE OF INTIMACY AND INTIMATE RELATIONSHIPS IN ASSISTED LIVING}

\section{A. Fitzroy, C.L. Kemp, E.O. Burgess, Georgia State}

University, Atlanta, Georgia

Intimate relationships are rarely studied in the context of older adults' care arrangements, particularly in long-term care settings. Consequently, little is known about the availability and effects of intimacy in the lives of long-term care residents. Addressing this gap, this paper focuses on the intimacy experiences of older adults residing in 8 diverse assisted living (AL) communities. We analyze data drawn from a fiveyear NIA-funded qualitative study (R01AG044368) guided by the "Convoys of Care" model. Previous analysis of study data shows that most residents experience some form of intimacy in their day-to-day lives. Yet, a range of multilevel factors influences intimacy and many have unmet intimacy needs in these settings. Building on this work, our aim is to understand how the presence and absence of intimacy influences residents' lives, relationships, and care experiences in AL. Findings show that some AL residents sought out, created, and maintained intimacy through a variety of interaction and relationships and with other residents, family, and care staff. Residents who had companionship, particularly involving physical interactions, felt it enhanced their quality of life and care. In some instances, such companionship created conflict within care networks. Mostly, however, formal and informal caregivers experienced relief and satisfaction when residents established intimate connections, including friendships. Many residents desired, but lacked outlets for intimacy, which frequently led to feelings of loneliness, isolation, and sadness. This outcomes was particularly prevalent among men. Our findings imply the need for a holistic model of care that takes into account and addresses intimacy needs.

\section{SYNERGISTIC IMPACTS OF PRE-HOMEBOUND AND SOCIAL ISOLATION ON MORTALITY AMONG THE OLDER IN JAPAN}

M. Yasunaga ${ }^{1}$, M. Nishi ${ }^{1}$, M. Hasebe ${ }^{2}$, K. Nonaka ${ }^{1}$, T. Koike ${ }^{3}$, H. Suzuki ${ }^{1}$, Y. Murayama ${ }^{1}$, Y. Fujiwara ${ }^{1}$, 1. Tokyo Metropolitan Institute of Gerontology, Itabashi-ku, Tokyo, Japan, 2. Sei-Gakuin University, Saitama, Japan, 3. Kyushu Sangyo University, Fukuoka, Fukuoka, Japan 